\title{
The Feedforward Method of Controlling Three-Level Diode Clamped Converters with Small DC-Link Capacitors
}

\author{
Nikola Celanovic, Ivan Celanovic ${ }^{*}$, Dushan Boroyevich ${ }^{* *}$ \\ ABB Corporate Research Center LTD \\ Power Electronic Systems CHCRC.S1 \\ CH-5405 Baden-Dättwil, Switzerland \\ nikola.celanovic@ch.abb.com
}

\author{
${ }^{* *}$ Center for Power Electronic Systems (CPES) \\ The Bradley Department of Electrical and Computer Engineering \\ Virginia Polytechnic Institute and State University \\ 657 Whittemore Hall, Blacksburg, VA 24060-0111 \\ dushan@vt.edu
}

\begin{abstract}
This paper introduces a control method, which enables a distortion-free operation of a three-level converter in the presence of the significant neutral point voltage ripple. Application of this control method allows a trade-off between voltage capacity of the switching devices and the size of the dc-link capacitors.
\end{abstract}

\section{INTRODUCTION}

The size of the dc-link capacitors for almost any dc/ac three phase converter is determined by either their energy storage function, which is often the case for motor drive applications, or with the switching frequency ripple filtering function. For three-level converters that do not have the energy storage requirement of some of the drive systems it is not the switching frequency ripple that determines the size of the dc-link capacitors but the third harmonic neutral point (NP) ripple current. This ripple current can for certain loading conditions be very large and therefore significantly larger dc-link capacitors are required than the ones required for filtering the switching frequency ripple only.

However, by introducing a feedforward algorithm that can prevent ripple in the NP from creating distortion in the ac waveforms it might be feasible to operate the converter with the significant ripple in the NP provided that the switching devices have the necessary voltage margin.

\section{THE EFFECT OF THE VOLTAGE ERROR IN THE NP}

Fig. 1 shows the functional diagram of the three-level voltage source converter, where each phase leg is represented by single-pole triple-throw switch. Each switch can assume one of the three states $S=\{0,1,2\}$. Combination of switching states of a three-level converter from Fig. 1 produces well defined three phase line voltages, which can be represented as two-dimensional voltage space vectors. In case the error in NP voltage $\Delta \mathrm{V}$ is zero ( $V_{p}=V_{n}=V_{d d} / 2$ ), the switching vectors can be represented as in Fig. 2.

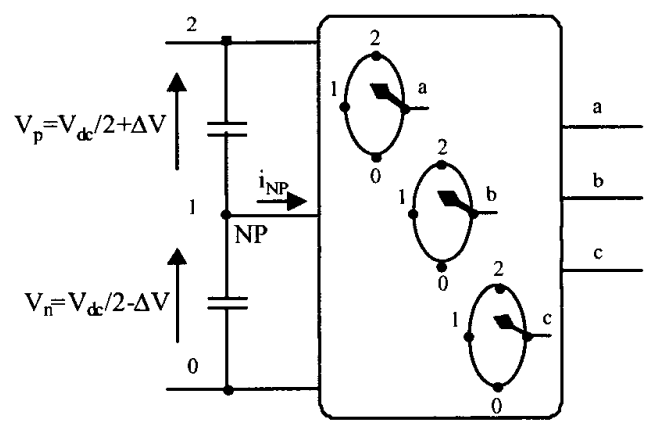

Fig. 1. Functional diagram of a three-level converter.

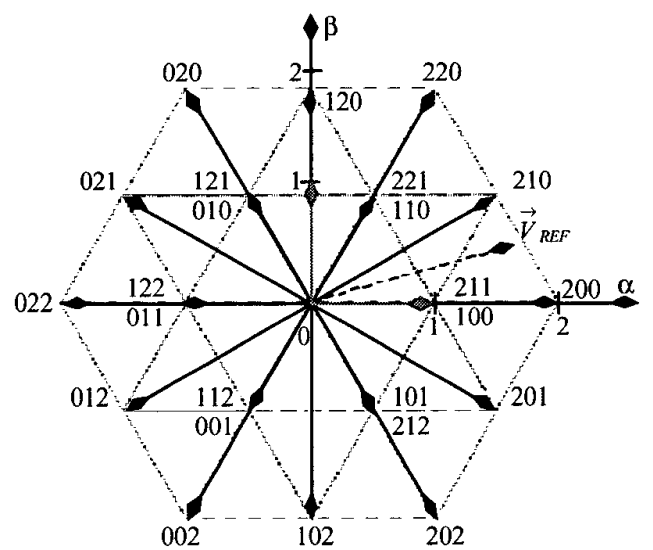

Fig. 2. Voltage space vectors of a three-level converter.

This method was developed with the generous help and support from the engineers of the General Electric Corporate Research Center in Schenectady, NY. All rights are the property of General Electric. 
However, if there is a significant voltage ripple present in the NP, as can be the case for certain transient conditions or in the case of purposely-reduced size of the dc-link capacitors, some of the vectors change their locations. Large voltage space vectors $(200,220,020$, $022,002,202)$ do not connect any of the phases to the NP and they remain unaffected by the voltage error in the NP. For example, in case $\Delta \mathrm{V}<0$, the medium vectors $(210,120$, $021,012,102,201)$ shift like in Fig. 3, while the small vectors "split" like in Fig. 4.

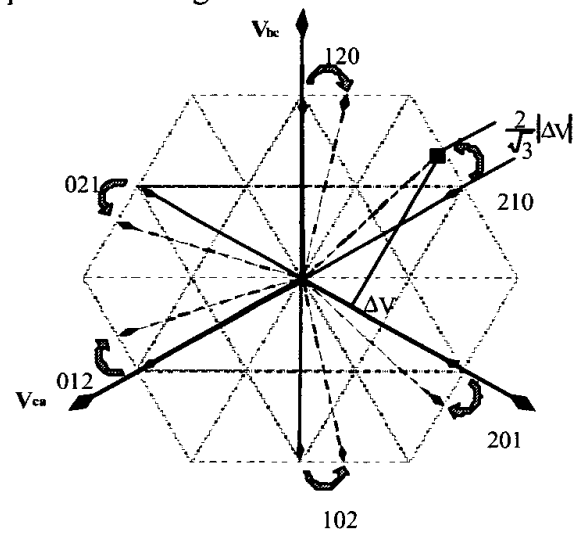

Fig. 3. Shift of the medium voltage vectors due to the $\Delta \mathrm{V}>0$ error in NP voltage.

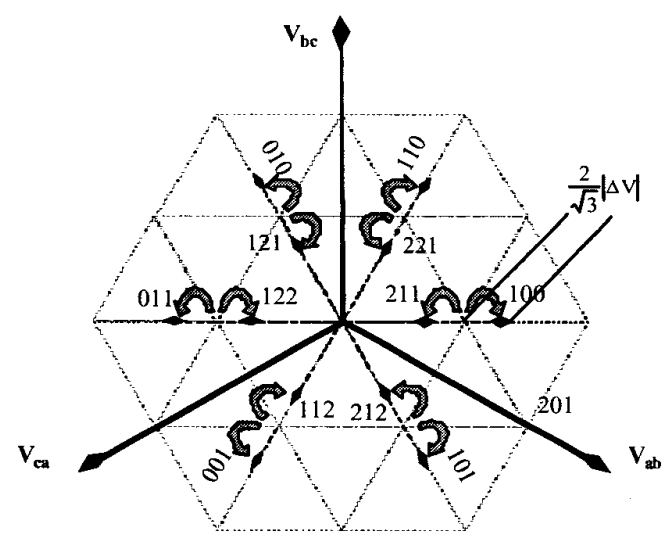

Fig. 4. "Splitting" of the small voltage vectors due to the $\Delta \mathrm{V}>0$ error in NP voltage.

Fig. 5 shows significant distortion in the line output voltage waveforms in the presence of a large NP voltage ripple for inverter and rectifier mode of operation respectively. This gives strong motivation for developing feedforward algorithm to alleviate this problem.

Because the large vectors remain unaffected by the disturbance in the mid-point voltage, the maximum amplitude of the reference vector that can be synthesized remains unaffected. Intuitively, this was to be expected considering the fact that three-level converter can always operate as two-level one which has no NP related issues.
This also indicates that it should be possible to compensate for the effect of the NP voltage ripple without sacrificing the converter's voltage gain.
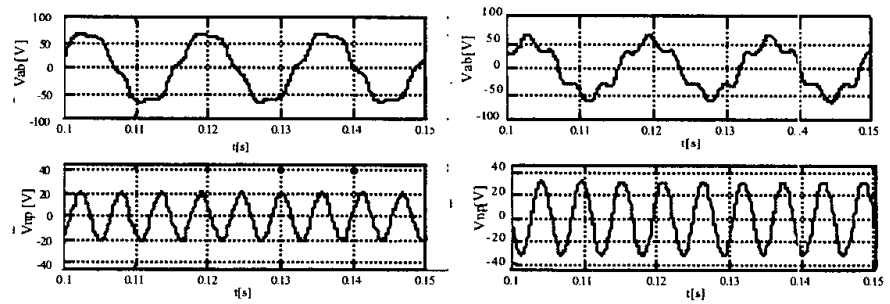

Fig. 5. The effect of the NP voltage ripple on output voltage waveforms.

\section{THE FEEDFORWARD ALGORITHM}

The feedforward algorithm proposed in this paper can be implemented for both space vector and carrier based modulation, but for the reasons of simplicity and more intuitive treatment only the carrier-based version of feedforward algorithm will be presented and studied.

\section{A. The Feedforward Algorithm}

Fig. 6. Shows the block diagram of the three-level NPC converter with feedforward controller. The idea behind the feed forward algorithm is to measure the DC link voltage unbalance and to modify the amplitude of the triangular carrier waveforms so that the ratio of the upper and the lower carrier corresponds to the ratio of upper and lower dc-link capacitor voltage.

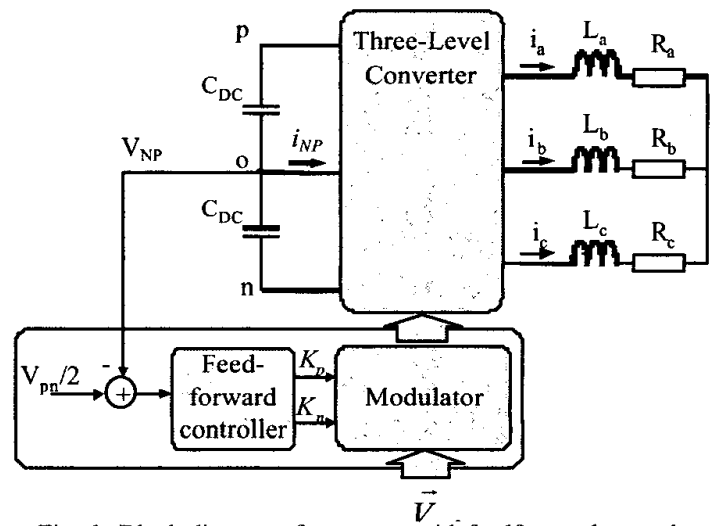

Fig. 6. Block diagram of converter with feedforward control.

One way to satisfy this requirement is to continuously adjust the amplitude of the triangular carrier waveforms such that:

$$
K_{p}=\frac{V_{p}}{V_{d c} / 2}, \quad K_{n}=\frac{V_{n}}{V_{d c} / 2},
$$

where $K_{p}$ and $K_{n}$ are the peak-to-peak values of the top and bottom carrier waveforms, respectively. 

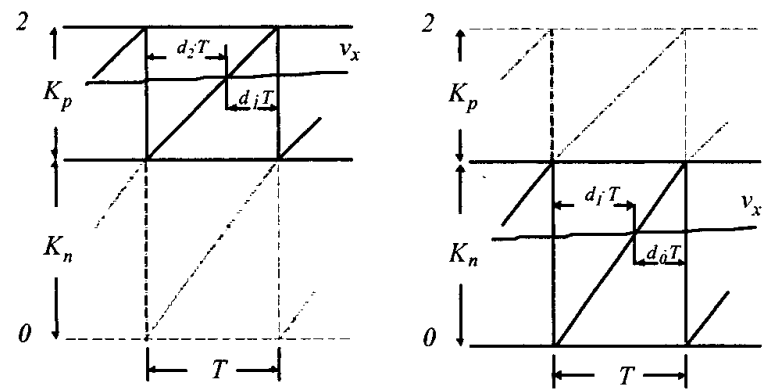

Fig. 7. Duty cycles for the sine triangle three-level modulation with feed forward control.

Assuming that carrier frequency is much larger than the fundamental frequency of the modulating waveform and considering Fig. 7 , it can be shown that the average duty cycles for any phase are given by

$$
d_{2 x}=\frac{v_{x}-K_{n}}{K_{p}} \quad d_{1 x}=\frac{2-v_{x}}{K_{p}}, \text { for } v_{x}>K_{n}
$$

and

$$
d_{x l}=\frac{v_{x}}{K_{n}} \quad d_{0 x}=\frac{K_{n}-v_{x}}{K_{n}}, \text { for } v_{x}<K_{n}
$$

where $x \in\{a, b, c\}$ and $d_{y x}$ where $y \in\{0,1,2\}$ represents the switching cycle averaged part of the switching period the phase $x$ is connected to the top rail $(y=2)$, the NP $(y=1)$ and the bottom rail $(y=0)$. Modulating waveform $v_{x}$ for any phase is given as:

$$
v_{x}=A \cdot \sin \left(\omega t+\phi_{x}\right)+K_{n}+\ddot{a}
$$

where $v_{x} \in[0,2], A \in[0,1]$ and $A<K_{n}+\delta<2-A$, and $\delta$ is the zero sequence component which is used to control the voltage balance in the NP as explained in [1].

\section{B. The Continuity Study}

From the development so far it might not be clear whether there exists a discontinuity in the average value of the output signal when the modulating signal crosses the "moving" boundary between upper and lower carrier signal. The effect of crossing the "moving" boundary can perhaps best be examined by writing equations for the average output voltage for the cases when $v_{x}>K_{n}$, and $v_{x}<K_{n}$.

Using equations (1) and (2) the average phase to NP output voltage for $v_{x}>K_{n}$ is

$$
v_{x 0}=d_{2} \cdot V_{p}+d_{1} \cdot 0=\left(v_{x}-K_{n}\right) \cdot \frac{V_{d c}}{2} .
$$

Similarly for $v_{x}<K_{n}$ using equations (1) and (3) the average output voltage is

$$
v_{x 0}=d_{1} \cdot 0-d_{0} \cdot V_{n}=\left(v_{x}-K_{n}\right) \cdot \frac{V_{d c}}{2},
$$

which is exactly the same expression as (5). Therefore, there are no discontinuities at the boundary of the upper and lower carrier waveform for the feedforward algorithm.

\section{The Stability Analysis of The Algorithm.}

Adding the zero sequence, $\delta$ into the modulating signal controls the NP voltage balance in the three-level converter. Clearly, the feedforward term also influences the zero sequence of the modulating signal and therefore the control of the NP. Fig. 8. shows the situation with the feedforward compensation of a large unbalance between the two dc-link capacitor where the zero sequence control of the NP is significantly limited due to the large amplitude of the modulating signal.

Because of the feedforward action more power will be exchanged between the upper dc-link capacitor and the converter's ac side than between the lower dc-link capacitor and the converter's ac-side. Therefore, when the converter is operating in the inverter mode the capacitor with the higher voltage will always tend to discharge, which helps stabilize the NP. In the case of the rectifier mode of operation the power flows in the opposite direction and tends to further increase the voltage of the capacitor with the higher voltage.

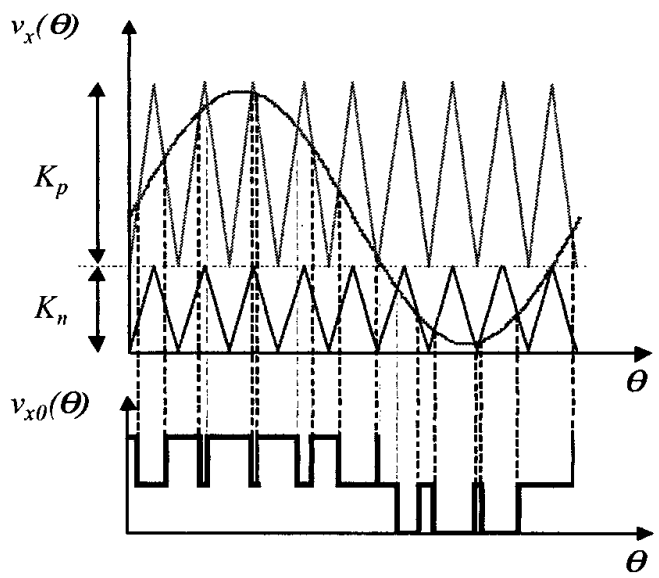

Fig. 8. The PWM signal with strong feedforward component.

Fortunately, in the rectifier mode of operation the situations is not as bad as it may appear. In other words it is much easier to keep the average NP current zero in the presence of the significant third harmonic ripple than it is to keep it zero for the case of unbalanced constant dc-sources which is the case qualitatively analyzed in Fig. 8 due to its simplicity.

In fact, it will be shown that only under excessive ripple and the large power factor (PFC rectifier mode of operation) the NP can not be controlled. In that case the feedforward term has to be reduced to allow enough margin for the zero sequence to resume control of the NP. 


\section{NP CONTROL WITH FEEDFORWARD ALGORITHM}

In order to quantitatively study the influence of feedforward control on the control of the NP voltage control dynamics a line cycle average model is developed next. Fig. 9 shows one way how the effect of the converter on the NP can be modeled. Clearly the NP voltage depends on the neutral point current, which depends on the load currents and the NP phase duty cycles $d_{l a}, d_{l b}, d_{l c}$, as in (8).

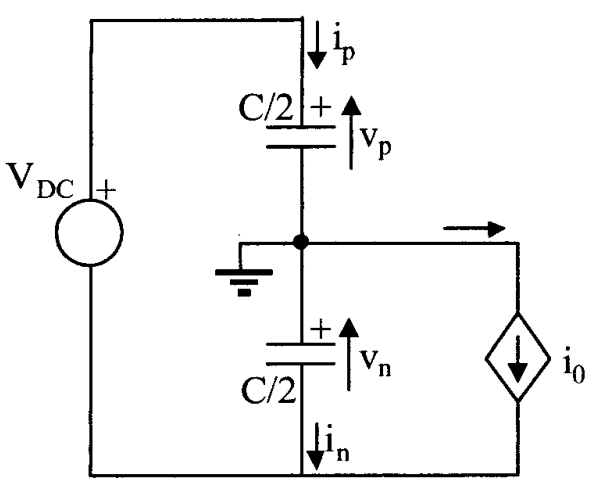

Fig. 9. The model of the modulator with respect to the NP current.

$$
C \frac{d v_{p}}{d t}=d_{l a} \cdot i_{a}+d_{l b} \cdot i_{b}+d_{l c} \cdot i_{c}
$$

Averaging the right hand side of the equation (7) shows the influence of the feedforward control to the average current in the NP:

$$
\begin{aligned}
\bar{i}_{N P} & =\frac{1}{2 \pi} \int_{0}^{2 \pi}\left(d_{l a} \cdot i_{a}+d_{l a} \cdot i_{b}+d_{l c} \cdot i_{c}\right) d \grave{e}= \\
& =\frac{3}{2 \pi} \int_{0}^{2 \pi}\left(d_{l a} \cdot i_{a}\right) d \grave{e}
\end{aligned}
$$

In order to perform the line-cycle averaging, boundary between the piece wise continuous duty cycles needs to be found. From Fig. 10 the first discontinuity in the duty cycle comes from the condition,

$$
K_{n}+\delta+A \cdot \sin (\alpha)=K_{n}
$$

Where the limits for integration can be chosen as

$$
\alpha=-\sin ^{-1} \frac{\delta}{A}, \quad \beta=\pi-\alpha \text { and } \gamma=2 \cdot \pi+\alpha
$$

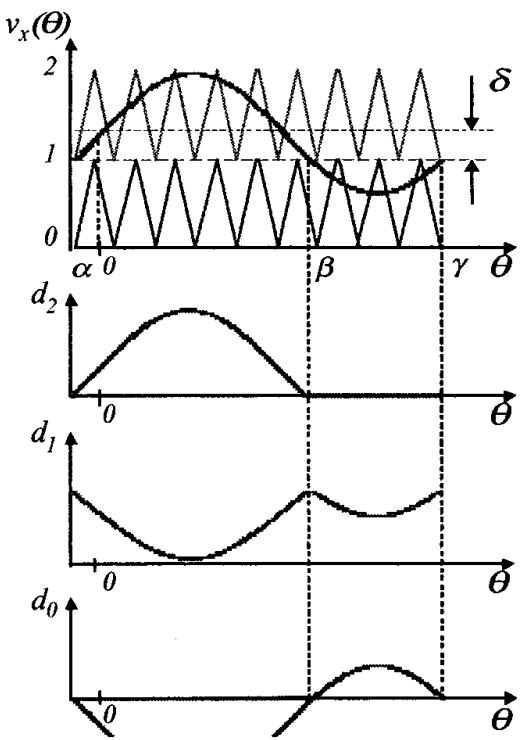

Fig. 10. The duty cycles of the sine triangle modulator with zero sequence.

By substituting the modulating signal from (4) into the expressions for NP duty cycles $\left(d_{l}\right.$ in Equations (2) and (3)), the line cycle average of the NP current can be found by integrating,

$$
\begin{aligned}
\overline{i_{N P}}=\frac{3}{2 \pi} & \cdot\left[\int_{\dot{a}}^{\dot{b}} I \cdot \sin (\hat{e}+\ddot{\partial}) \cdot\left(\frac{\left.2-\left(K_{n}+\ddot{a}+A \sin \theta\right)\right)}{K_{p}}\right) \cdot d \grave{e}+\right. \\
& \left.+\int_{a}^{\dot{d}} I \cdot \sin (\hat{e}+\ddot{\partial}) \cdot\left(\frac{K_{n}+\ddot{a}+A \sin (\theta)}{K_{n}}\right) \cdot d \grave{e}\right]
\end{aligned}
$$

The resulting average current is

$$
\begin{aligned}
\overline{i_{N P}}= & \frac{-3 \cdot I \cdot \cos (\phi)}{4 \cdot \pi \cdot A \cdot K_{n} \cdot K_{p}} \cdot\left[2 \cdot\left(4 \cdot K_{n}+2 \cdot \delta-4\right) \cdot \sqrt{A^{2}-\delta^{2}}\right) \\
& -A^{2} \cdot \pi \cdot\left(K_{n}-K_{p}+2 \cdot A^{2} \cdot\left(K_{p}+K_{n}\right) \cdot \sin ^{-1}\left(\frac{\delta}{A}\right)\right]
\end{aligned}
$$

valid for

$$
A<K_{n}+\delta<2-A \text {, }
$$

which makes the average value of NP current

$$
\overline{i_{N P}}=-K\left(A \delta, K_{p}, K_{n}\right) \cdot I \cdot \cos (\varphi),
$$

where 


$$
\begin{aligned}
& K\left(A \delta, K_{p}, K_{n}\right)=\frac{3}{4 \pi A K_{n} K_{p}}\left[2\left(4 K_{n}+2 \delta-4\right) .\right. \\
& \sqrt{A^{2}-\delta^{2}}-A^{2} \pi\left(K_{n}-K_{p}+2 A^{2}\left(K_{p}+K_{n}\right) \sin ^{-1}\left(\frac{\delta}{A}\right)\right.
\end{aligned}
$$

Indeed $K\left(A, \delta K_{p}, K_{n}\right)$ determines how much current flows in or out of the NP as function of $\delta$ NP voltage error and modulation index as shown in Fig. 11. Flat part of the curve represents the overmodulation boundary.

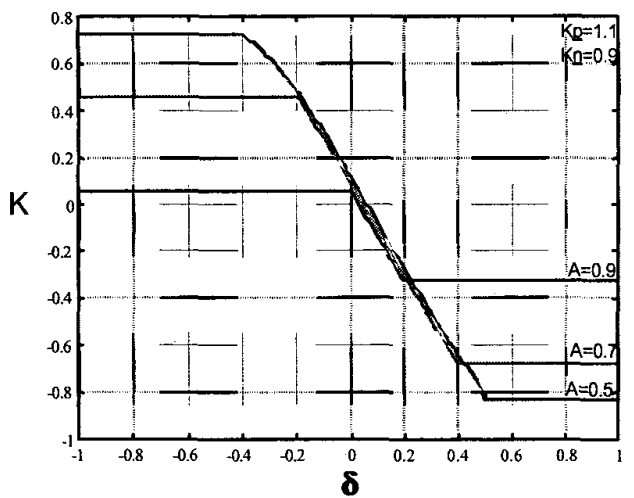

Fig. 11. $\mathrm{K}$ as function of $\delta$ for different modulation index, $\mathrm{A}$ and for $K_{p}=1.1$ and $K_{n}=0.9$.

Outside the region (13) the modulator works in the overmodulation mode (flat part of the curve in Fig. 11.), a mode that is not covered in this analysis. Otherwise, quite expectedly in case the dc-link capacitor charges are balanced, $K_{p}=K_{n}=1$, the equation (12) simplifies to the expression for the average NP current for the case with no feedforward terms,

$$
\overline{i_{N P}}=\frac{-3 \cdot I}{\pi \cdot A} \cos (\phi)\left[\delta \cdot \sqrt{A^{2}-\delta^{2}}+A^{2} \cdot \sin ^{-I} \frac{\delta}{A}\right]
$$

In order to obtain small-signal model for NP voltage dynamics in the presence of feedforward control, the equation (14) can be linearized around the operating point ( $\delta=0, K_{p}=K_{n}=1$ ), which results in the same linear model as the one derived in [2],

$$
\overline{i_{N P}}=-\frac{6}{\pi} \cdot I \cdot \cos (\phi) \cdot \delta
$$

Therefore around the operating point, that quite realistically assumes the balanced charge in the dc-link capacitors, and the zero sequence voltage equal to zero, the small signal average current model is the same as the small signal model of the modulator without feedforward control of NP. This indicates that the feedforward algorithm should not affect the NP control strategy.

\section{SIMULATION AND EXPERIMENTAL RESULTS}

Finally, through the experiment and simulation a quick analysis of the validity of the NP controller model will be provided together with the analysis of the stability of the NP control in rectifier mode with zero average current but large third harmonic component.

\section{Analysis of the line cycle average model of the feed forward algorithm}

The line cycle average method of analysis leaves the impression that the effect of the modulator is nothing other than a current gain. Taking into account approximations such as the line cycle averaging and linerization in the presence of the ripple that is not small it might be necessary to verify the validity of those assumptions experimentally.

Fig. 12 shows the functional diagram of the experiment which consisted of a three-level neutral point clamped converter, with the modulator implemented using the fast space vector modulation algorithm [4]. The converter was operating in the inverter mode with the $80 \mathrm{~V}$ dc-link voltage and with the RL load $(\mathrm{R}=.72 \Omega, \mathrm{L}=1.8 \mathrm{mH})$. Fig. 13 shows the controller loop gain for different modulation index (output voltage, and output current) of the converter. The loop gain measurement results show only the first order response with the phase slowly increasing as the consequence of the delay in the control loop. The first order response can be observed for other power factor loads as well. This justifies the assumption that the converter can be modeled as a nonlinear gain.

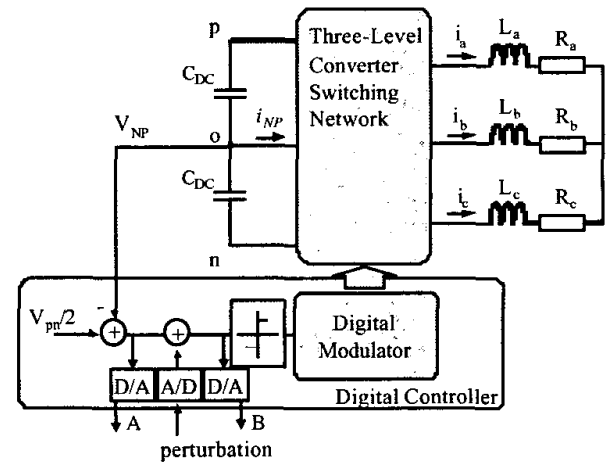

Fig. 12. Experimental measurement of the loop gain in the NP controller.

\section{E. Analysis of the switching cycle average model of the feed forward algorithm}

The average model of the converter based on the Equations (1) and (2) was developed to investigate the stability properties of the NP control in case of zero average NP voltage but a very large voltage ripple. The model was first compared with the experimental results for the $R L$ load with $R=0.72 \Omega, L=1.8 \mathrm{mH}$ and the dc-link of $80 \mathrm{~V}$, Fig. 14 . The results show very good match between the simulation and the measurement. 

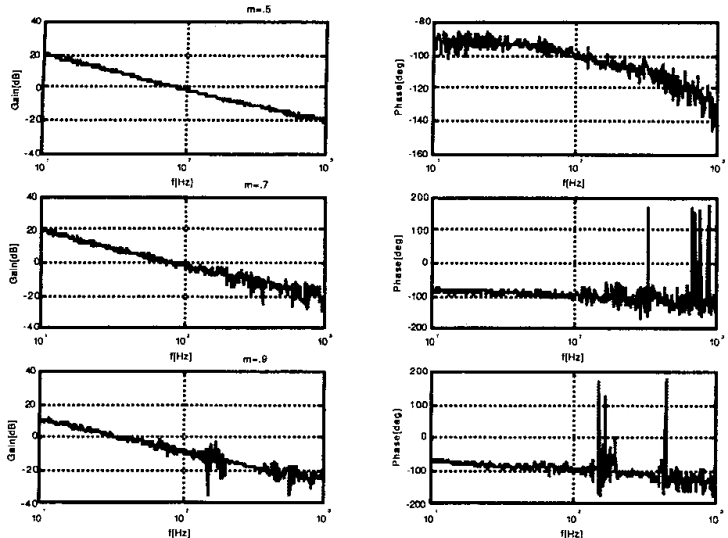

Fig. 13. Measurement of the NP control loop gain.
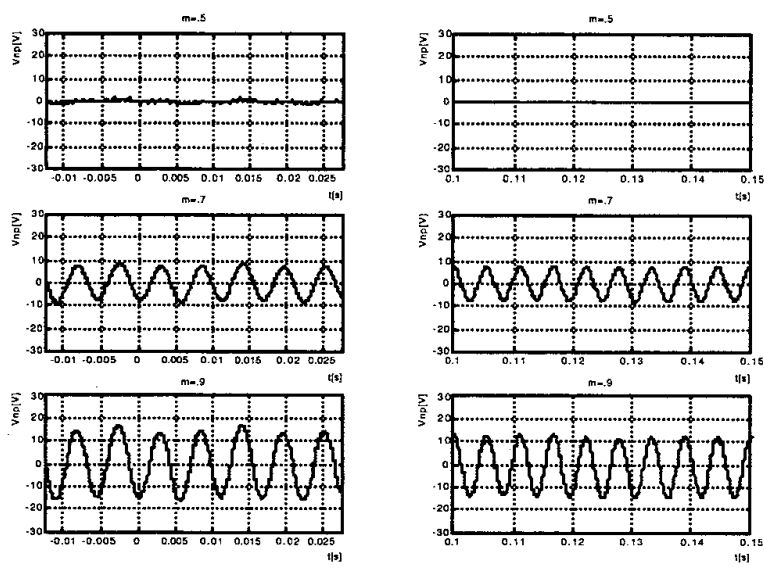

Fig. 14. Measured and simulated NP ripple of a three-level converter.

Finally the stability of the NP control was studied based on the line cycle average. The study showed that the influence of the feedforward in the rectifier mode causes instability only in the extreme cases with very large current in the NP voltage ripple and the small values of the power factor angles. This is not of too much concern because for higher power factor current the ripple is always smaller than for the lower power factor. In addition due to the devices limitations it might not be feasible to run the converter with the NP voltage ripple approaching half of the total dc-link like in Fig. 15.

\section{CONCLUSIONS}

This paper introduces and analyzes the feedforward algorithm as a method that can successfully compensate any size of the voltage ripple in the NP and in that way allow the tradeoff between the voltage capacity of the switching devices and the size of the dc-link capacitors.

The influence of the new algorithm to the control of the NP is studied on the example of the sine triangle modulator. The influence of the modulator on the NP controller is modeled with the average models developed at the two different time scales. First at the fast scale, the time scale of the switching frequency, and than those results were used to develop an average model on the slow scale, the time scale of the line cycle average.
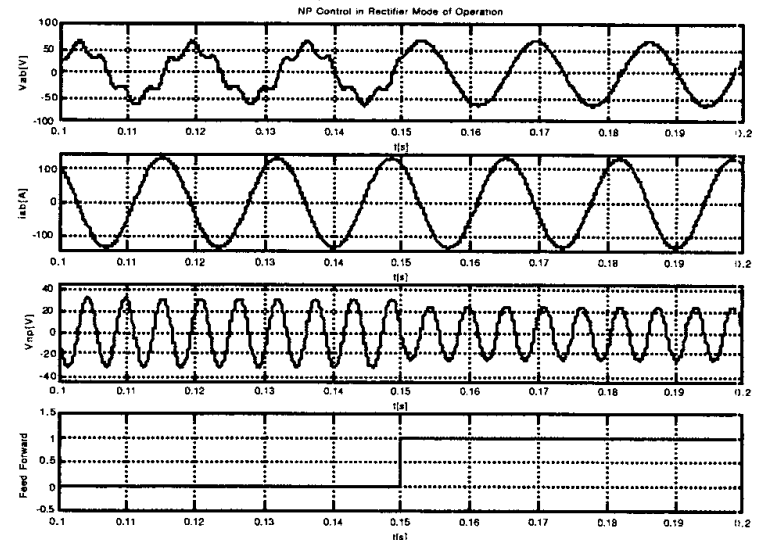

Fig. 15. Three phase converter in the rectifier mode of operation with $80 \mathrm{~V}$ dc-link voltage. Modulation index $\mathrm{m}=0.95$ and the current 85 degrees reactive power factor. The fedforward control is initially off.

1.) The line voltage without and than with the feedforward controller

2.) The line current

3.) The NP voltage ripple

4.) The feedforward command signal

The line cycle average model of the sine triangle modulators influence on the control of the NP is than linearized around the operating point and can be used to design a NP voltage controller. The simulation results show the effectiveness of the algorithm. However, some additional work is required to precisely determine the bounds for the stable operation in the rectifier mode of operation, even though the simulation studies show that if the amplitude of NP voltage ripple does not exceed $15 \%$ of the dc-link voltage $\left(V_{p n}\right)$ the system can maintain the stability of the NP, for any current power factor.

\section{ACKNOWLEDGMENT}

The author wishes to acknowledge the invaluable contributions made to this work by J. P. Lyons, P. Szcsesny and V. Vlatkovic (all with GE Corporate Research Center in Schenctady NY), and to thank them for their help and support.

\section{REFERENCES}

[1] S. Ogasawara, H. Akagi, "Analysis of Variation of Neutral point Potential in Neutral-Point-Clamped Voltage Source PWM Inverters," IEEE-IAS Conference Record, 1993, pp. 965-970.

[2] C. Newton, M. Sumner, "Neutral Point Control for Multi-Level Inverters: Theory, Design and operational limitations," IEEE-IAS Conference Record, 1997, pp. 1336-1343.

[3] N. Celanovic, D. Boroyevich, "A Comprehensive Study of Neutral-Point Voltage Balancing Problem in Three Level Neutral-Point-Clamped Voltage Source PWM Inverters", IEEE Transactions on Power Electronics, vol. 15, no. 2, March 2000, pp. 242-249.

[4] N. Celanovic, D. Boroyevich, "A Fast Space Vector Modulation Algorithm for Multilevel Three-Phase Converters," IEEE Transactions on Industry Applications, vol. 37, no. 2, March/April 2001, pp. 637-641. 